Forum 2021 · 36:396-399

https://doi.org/10.1007/s12312-021-00989-y

Online publiziert: 14 . September 2021

○) Springer Medizin Verlag $\mathrm{GmbH}$, ein Teil von

Springer Nature 2021

HAMBURGER

:::::: KREBSGESELLSCHAFT E.V.

\title{
Ambulante Krebsberatung in Zeiten von Corona
}

\author{
Zahlen, Daten und subjektive Eindrücke aus der Hamburger \\ Krebsgesellschaft e.V.
}

\author{
Franziska Holz · Monika Bohrmann² \\ ' Geschäftsführung und Krebsberatung, Hamburger Krebsgesellschaft e.V., Hamburg, Deutschland \\ ${ }^{2}$ Leitung Beratungsdienst, Hamburger Krebsgesellschaft e. V., Hamburg, Deutschland
}

\section{Zusammenfassung}

\section{In diesem Beitrag}

- Zusätzliche Herausforderungen

- Beratung 2.0

- Daten und Fakten

- Psychosoziale Dimensionen

- Und in Zukunft?
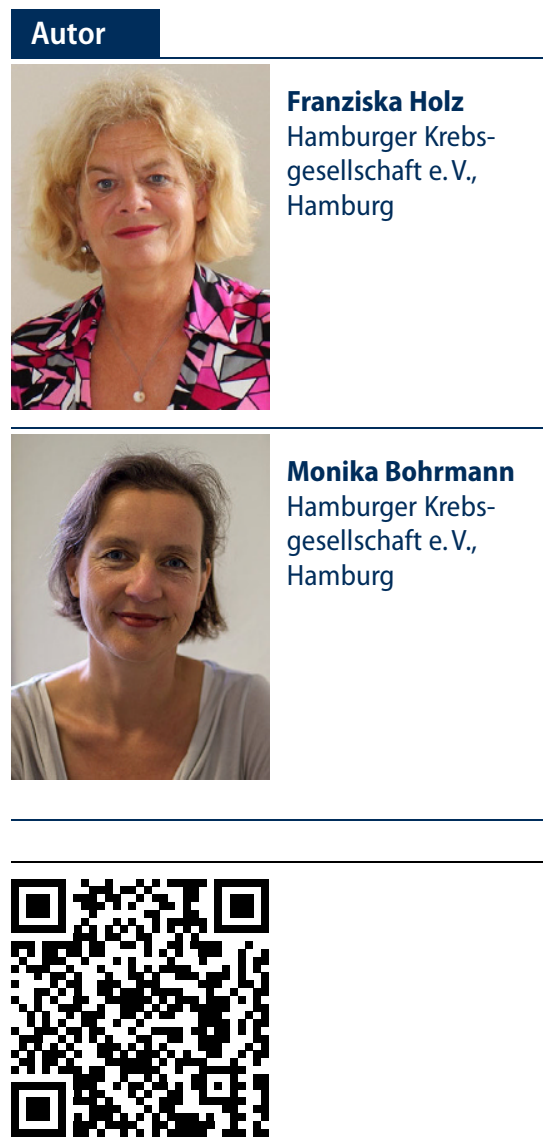

QR-Code scannen \& Beitrag online lesen

Menschen mit einer Krebserkrankung stehen durch das Coronavirus vor besonderen Herausforderungen. Krebspatient*innen gehören zu den Risikogruppen und müssen verstärkt vor einer COVID-19-Infektion geschützt werden. Während der Pandemie hatten an Krebs Erkrankte und Angehörige hohen Beratungsbedarf. Landesweit gab es viele interessante neue und konstruktive Lösungen, die durch eine große Flexibilität aller Beteiligten, höhere Investitionen der Vereine und das schnelle und kompetente Agieren in den Landeskrebsgesellschaften ermöglicht wurden. Dadurch konnten die für die Betroffenen so wichtigen Beratungsleistungen und begleitenden Angebote, gerade in Zeiten, in denen die klinisch-stationären und sonstigen Versorgungsangebote für Menschen mit Krebs massiv eingeschränkt waren, durchgehendend aufrechterhalten werden. Sicher werden einige der entstandenen Formate und Projekte auch in Zukunft Bestand haben. So hat sich das Angebot der Videoberatungen auch in der ambulanten Krebsberatung etabliert.

\section{Schlüsselwörter}

COVID-19-Pandemie · Ambulante Versorgung · Fernberatung · Psychoonkologie · Onlineunterstützung

Seit der ersten COVID-19-Pandemiewelle zu Beginn des letzten Jahres kam es immer wieder zu Einschränkungen, die v.a. die Schwächsten in der Gesellschaft besonders getroffen haben.

Um mögliche Auswirkungen der Pandemie auf die onkologische Versorgung schnell zu erfassen, gründeten die Deutsche Krebsgesellschaft, die Deutsche Krebshilfe und das Deutsche Krebsforschungszentrum eine Taskforce und baten 18 Comprehensive Cancer Centers, über einen Zeitraum von 5 Monaten die Versorgungslage von onkologischen Patienten zu erfassen. Über $90 \%$ der befragten onkologischen Zentren gaben an, dass es zu Veränderungen bei der Nachsorge und in den abgefragten Bereichen "Psychoonkologie", "Ernährungs- und Bewegungstherapien“ und "soziale Beratung" gekommen sei. Da diese Themenbereiche allesamt schwerpunktmäßig von ambulanten Krebsberatungsstellen abgedeckt werden, schildern wir im Folgenden die Auswirkungen der Pandemie für onkologisch Erkrankte in der psychosozialen Krebsberatung aus Sicht der Hamburger Krebsgesellschaft e.V.

\section{Zusätzliche Herausforderungen}

Menschen mit einer Krebserkrankung stehen durch das neuartige Coronavirus vor besonderen Herausforderungen. Krebspatient*innen gehören zu den Risikogruppen und müssen verstärkt vor einer Infektion geschützt werden. Das bedeutete für die allermeisten an Krebs Erkrankten ein Leben in "freiwilliger" Quarantäne über mehrere Wochen und Monate. Der Austausch mit Familie, Freund*innen und Bekannten war für über 1 Jahr - bis auch den Krebser- 


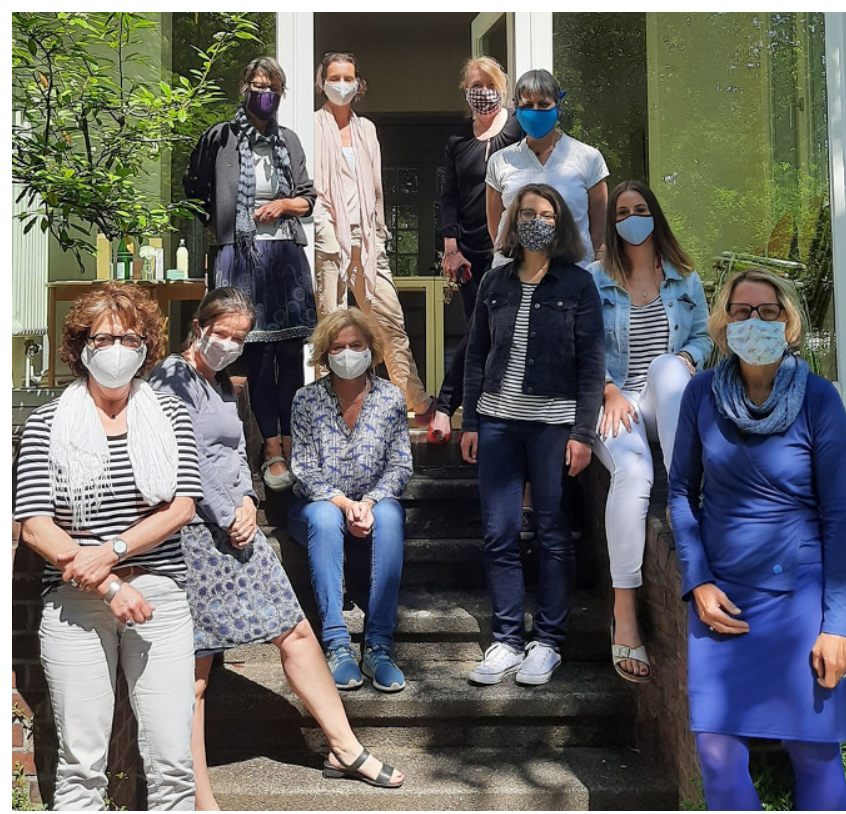

Abb. $1<$ Das Team der Hamburger Krebsgesellschaft in Zeiten von Corona, Juni 2020. (Hamburger Krebsgesellschaft privat)

krankten aus der Priorisierungsgruppe 2 im April 2021 endlich ein Impfangebot gemacht werden konnte - stark limitiert und meist auf Telefon- oder Videokontakte beschränkt. Viele unserer Ratsuchenden haben den Verlust von Nähe zu anderen Menschen und das Gefühl der Einsamkeit als sehr schmerzhaft erlebt. Aber auch etliche kulturelle Ablenkungen sind während des Lockdowns ersatzlos weggefallen. Die daraus resultierende Isolation führte bei vielen Betroffenen zu einem erhöhten Gesprächs- und Beratungsbedarf.

Nach dem ersten Erlass der Coronaverordnung des Hamburger Senats vom 16.03.2020 gab es auch für die Hamburger Krebsgesellschaft nur die Option, alle persönlichen Kontakte zu den Ratsuchenden sofort einzustellen.

\section{Beratung 2.0}

Die Mitarbeiterinnen der Hamburger Krebsgesellschaft ( Abb. 1) haben rasch und flexibel reagiert. Zwar musste das Kursangebot vorläufig komplett gestrichen werden, die medizinischen, psychoonkologischen und sozialrechtlichen Beratungen liefen dennoch unvermindert weiter, zunächst ausschließlich per Telefon. „Das Signal, das wir unbedingt sofort für unsere Ratsuchenden aussenden wollten, lautete: Wir sind auch jetzt für euch da! Denn vermutlich brauchten uns die Menschen in dieser besonderen
Zeit mehr denn je", berichtet Geschäftsführerin Franziska Holz. Dennoch stellten Veränderungen der Arbeitsstrukturen, die häufig wechselnden und widersprüchlichen Verordnungen und die laufenden Aktualisierungen der Hygienekonzepte sowie, last but not least, die technischen Herausforderungen durch neue Formate (Videoberatung, Telefonkonferenzen) und Arbeitsbereiche (Homeoffice) nicht nur das Team, sondern auch die Leitungskräfte vor besondere Aufgaben. Ganz zu schweigen von den zeitlichen und monetären Belastungen: Kauf überteuerte Masken und Selbsttests sowie großer Mengen an Reinigungs- und Desinfektionsmitteln, die, anders als in anderen Einrichtungen der Gesundheitsversorgung, von den Krebsberatungsstellen ausschließlich selbst finanziert werden mussten.

Der Gesprächsbedarf war immens - unsere Drähte liefen heiß! Waren es zu Beginn der Krise noch viele medizinische Fragen, die sich auf das neuartige Virus und die damit verbundenen Infektionsrisiken und Schutzmaßnahmen bezogen, bereiteten etlichen Erkrankten im weiteren Verlauf eher die verschobenen oder veränderten medizinischen Behandlungen große Sorgen, ebenso wie die hohe Ansteckungsgefahr durch ein geschwächtes Immunsystem. Aber auch die Furcht, sich in der Klinik mit dem Coronavirus anzustecken, beschäftigte viele unserer Anrufer*innen. Im Frühjahr 2021 galten die meisten Fra- gen der Coronaimpfung von Krebskranken und Bezugspersonen. An die Hundert Anfragen, manche auch aus anderen Bundesländern, erreichten unsere Expert*innen.

\section{Daten und Fakten}

In Zahlen stellen sich die Beratungsleistungen der Hamburger Krebsgesellschaft e. V. wie nachfolgend beschrieben dar.

Die Auswertung der Stammdatensätze zeigte für die Jahre 2019 bis 2020 eine gleichbleibende Verteilung für Alter, Geschlecht, Status oder Art der Krebserkrankung. Im Vergleichsjahr 2019 waren, wie in den Vorjahren auch, die telefonischen und persönlichen Beratungen vor Ort etwa gleichmäßig verteilt. Die Gesamtstatistik für das Jahr 2020 zeigt bereits eine Verschiebung hin zu $65 \%$ Telefon- und nur noch $25 \%$ "Face-to-face-Kontakten", ebenso einen Anstieg der schriftlichen Beratungen (v.a. per E-Mail) auf fast $10 \%$. Mit genauerem Blick auf den ersten Lockdown (15.03.-15.06.2020) wird eine noch größere Umschichtung sichtbar: Hier lag der Schwerpunkt mit $83 \%$ deutlich auf den telefonischen Beratungen, während es nur $5 \%$ persönliche (in Einzel- und Ausnahmefällen) und $5 \%$ E-Mail-Beratungen gab. Ein ähnliches Bild ergibt sich für den zweiten Lockdown (15.12.2020-15.05.2021): In dieser Zeit waren es $86 \%$ Telefon- und $5 \%$ persönliche Beratungen. Videoberatungen, die im Sommer 2020 neu eingeführt wurden und erst seit $2021 \mathrm{im}$ Dokumentationssystem zu erfassen sind, nehmen in der ersten Jahreshälfte 2021 bereits einen Anteil von über $3 \%$ ein.

Von einem zurückhaltenden Nachfrageverhalten seitens der an Krebs Erkrankten während der Wochen mit sehr hohen Inzidenzzahlen können wir ebenso wie die Corona-Taskforce berichten. In jenen Hochphasen der Pandemie erfassten wir in unseren beiden Krebsberatungsstellen allerdings ein verstärktes Beratungsaufkommen durch hoch belastete Angehörige, die sich teils große Sorgen um ihre Familienmitglieder und Nächsten machten, die sie bedingt durch strikte Besuchsverbote in den Krankenhäusern während der gesamten Diagnostik- und Behandlungsphase in Klinik oder Praxis nicht begleiten durften. Die fehlende Beteiligung bei Gesprächen mit den Behandelnden und bei Therapie- 
entscheidungen führte zu großen Unsicherheiten und Informationsdefiziten bei An- und Zugehörigen. Fast unmöglich zu ertragen war es für nahe Angehörige immer dann, wenn ihre Liebsten allein und in völliger Isolation im Krankenhaus verstorben sind. In vielen dieser Fälle mussten unsere Beraterinnen intensivierte Trauerbegleitung leisten und mehr als einmal haben alle Beteiligten die Distanz von Video- oder Telefonverbindungen als viel zu groß erlebt.

\section{Psychosoziale Dimensionen}

Auch auf die sozialen und psychologischen Dimensionen hat der Pandemieverlauf Einfluss. Bei der Notwendigkeit, flexible tragfähige Absicherungs- und Versorgungslösungen zu finden und umzusetzen, war insbesondere die Sozialberatung gefragt. „Unsere Ratsuchenden beschäftigten v.a. Fragen, wie die Kinderund Haushaltsbetreuung in Coronazeiten zu regeln sind, aber auch Kündigungsdrohungen und Kurzarbeit waren häufige Beratungsanliegen", so Marile Woitschikowski, die als Sozialarbeiterin bei der Hamburger Krebsgesellschaft arbeitet. Speziell den Ratsuchenden, die die Sozialberatung wegen Antragstellungen oder Widerspruchsangelegenheiten in Anspruch genommen haben, ist der Wegfall der Präsenzberatung besonders schwergefallen, da Unterlagen, Anträge und Formulare nun nicht mehr gemeinsam von Antragstellenden und Beratenden ausgefüllt werden durften. Auch die Prüfung von Dokumenten erforderte bisweilen einen großen Erfindungsgeist aller Beteiligten.

Froh waren alle Beraterinnen, dass sie seit dem Wechsel zu einer cloudbasierten Dokumentationssoftware die erbrachten Beratungsleistungen nun problemlos auch im Homeoffice dokumentieren konnten.

Diplom-Psychologin Monika Bohrmann, die als Leiterin des Beratungsdienstes die psychoonkologische Beratungsarbeit verantwortet, sah ihre Aufgabe v.a. darin, die Ratsuchenden auch während des kompletten öffentlichen Rückzugs nicht allein zu lassen und diese dabei zu unterstützen, Spielräume zu entdecken, um gerade in dieser zusätzlich schwierigen Situation Kraft und Trost zu finden und Freude bringenden Aktivitäten nach-

Outpatient oncology counseling in times of corona. Figures, data, and subjective impressions from the Hamburg Cancer Society

People with cancer face particular challenges due to the coronavirus. Cancer patients belong to the risk groups and must be especially protected against infection with COVID-19. The counseling requirement of cancer patients and their relatives was high during the pandemic. Many interesting new and constructive solutions appeared throughout the country, made possible by a great flexibility of all involved parties, higher investments of beneficial associations, and the fast and competent acting of national cancer societies. As a result, the counseling and accompanying services-which are so important for affected persons-could be maintained throughout the period in which clinical inpatient care and other support services for cancer patients were massively restricted. Some of the resulting formats and projects will certainly continue to exist in the future. Thus, the offer of video consultations has also established itself in outpatient cancer counseling.

\section{Keywords}

COVID-19 pandemic · Outpatient care · Distance counseling $\cdot$ Psycho-oncology $\cdot$ Online social support

gehen zu können. Auch dazu haben wir gemeinsam mit unseren Kursleitungen einige kreative Ideen entwickelt und ein kleines, aber feines Onlinekursangebot auf die Beine gestellt, das unsere Klientel hoffentlich gut durch die ansonsten „kursund veranstaltungsfreie Zeit" gebracht hat. In den Sommermonaten haben wir komplett auf Freiluftaktivitäten umgestellt und so ganz neue Dimensionen erfahren dürfen.

Mit niedrigeren Inzidenzzahlen gab es immer wieder Lockerungen, selbst in der Hansestadt Hamburg, die in der Öffentlichkeit für strenge Coronaauflagen bekannt ist, und so haben wir in mehreren Phasen die Türen unserer beiden Krebsberatungsstellen mal geöffnet, mal wieder geschlossen. Wir nutzen in unseren Einrichtungen derzeit nur die großen Räume mit ausreichend Platz für persönliche Beratungen und haben ein abgestimmtes Wege-, Lüftungs- und Hygienekonzept erstellt. Außerdem herrscht in den Innenräumen der Beratungsstellen Maskenpflicht für das Beratungsteam und die Ratsuchenden; des Weiteren werden alle Kontakte lückenlos erfasst.

\section{Und in Zukunft?}

Sicher werden wir einige aus der Not entstandene Formate und Projekte auch in Zukunft beibehalten bzw. in neuer Form weiterführen. So wird das Angebot an Videoberatungen auf jeden Fall ausgebaut.
Für einige Kurse bringen die virtuellen Treffen sogar Vorteile für die Teilnehmenden mit sich, so können sich Angehörige beispielsweise leichter $90 \mathrm{~min}$ im häuslichen Arbeitszimmer vor den Computer setzen, als sich für 2-3 h von zu Hause zu entfernen oder sich von entfernten Wohn- oder Studienorten zuschalten. Auch die Teilnehmenden an Kursen der psychoedukativen Fatigue-Gruppe schätzen das „energiesparendere" Videoformat.

\section{Fazit}

Der Informationsfluss im Netzwerk der Landeskrebsgesellschaften hat auch in Pandemiezeiten vorbildlich funktioniert, den virtuellen Arbeitstreffen sei Dank. Die Landeskrebsgesellschaften haben sowohl Onlineformate als auch Hygienekonzepte ausgetauscht und sich über den Umgang mit Regularien gemeinsam mit den Verantwortlichen bei der Deutschen Krebsgesellschaft ins Benehmen gesetzt. Landesweit gab es viele interessante neue und konstruktive Lösungen, die durch eine große Flexibilität aller Beteiligten, erhöhte Investitionen der Vereine, das schnelle und kompetente Reagieren und ein vorrausschauendes Agieren der Vorstände in den 16 Landeskrebsgesellschaften ermöglicht wurden. Dadurch konnten die für die Betroffenen so wichtigen Beratungsleistungen und begleitenden Angebote, gerade in Zeiten, in denen die klinischstationären und sonstigen Versorgungs- 
angebote für Menschen mit Krebs und deren Angehörige massiv eingeschränkt waren, durchgehendend aufrechterhalten werden.

\section{Korrespondenzadresse}

\section{Franziska Holz}

Geschäftsführung und Krebsberatung, Hamburger Krebsgesellschaft e.V. Butenfeld 18, 22529 Hamburg, Deutschland f.holz@krebshamburg.de

\section{Monika Bohrmann}

Leitung Beratungsdienst, Hamburger Krebsgesellschaft e. V. Butenfeld 18, 22529 Hamburg, Deutschland m.bohrmann@krebshamburg.de

\section{Einhaltung ethischer Richtlinien}

Interessenkonflikt. F. Holz und M. Bohrmann geben an, dass kein Interessenkonflikt besteht.

Für diesen Beitrag wurden von den Autoren keine Studien an Menschen oder Tieren durchgeführt. Für die aufgeführten Studien gelten die jeweils dort angegebenen ethischen Richtlinien.

\section{Weiterführende Literatur}

1. Bundesministerium für Arbeit und Soziales (2020) SARS-CoV-2-Arbeitsschutzstandard, Version vom 16.04.2020 und ff

2. Deutscher Olympischer Sportbund Die zehn Leitplanken des Sports, Version vom 28.04.2020. https://www.dosb.de.Zugegriffen:30. Apr. 2020

3. Fröhling S, Arndt V (2020) Versorgung von Krebspatienten: Corona-Effekt in der Onkologie. Dtsch Arztebl 117(46):B-1893

4. Robert Koch-Institut (2020) COVID-19: Jetzt handeln, vorausschauend planen. StrategieErgänzung zu empfohlenen Infektionsschutzmaßnahmen und Zielen (2. Update). Epidemiol Bull 12:3-6. https://doi.org/10.25646/6540

5 . Ergänzung zum Beitrag „SARS-CoV-2: Informationen des Robert Koch-Instituts zu empfohlenen Infektionsschutzmaßnahmen und Zielen“ im Epidemiologischen Bulletin 7/2020 vom 13. Febr. 2020 und zu dem 1. Update im Internet vom 4. März 2020.

6. Diverse Informationen des Robert Koch-Instituts. https://www.rki.de.Zugegriffen:20.Aug. 2021

7. Verordnung zur Eindämmung der Ausbreitung des Coronavirus SARS-CoV-2 in der Freien und Hansestadt Hamburg (Hamburgische SARS-CoV-2-Eindämmungsverordnung HmbSARS-CoV-2-EindämmungsVO) in der jeweils gültigen Fassung. https://www.hamburg.de/ allgemeinverfuegungen/. Zugegriffen: 20. Aug. 2021

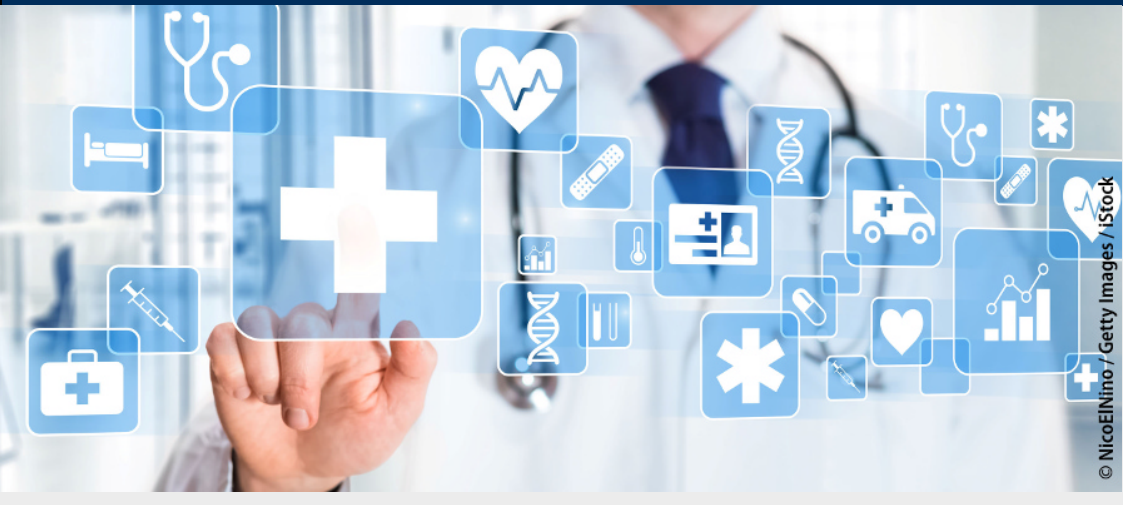

\section{DGIV-Bundeskongress, 29. Oktober 2020}

Überwindung der Sektorengrenzen - Erkenntnisse aus der CORONA-Krise

Die Deutsche Gesellschaft für Integrierte Versorgung e.V. ist ein deutschlandweit agierender Verein mit der Zielsetzung, die Integrierte Versorgung in der medizinischen, pflegerischen und sozialen Betreuung als Regelfall durchzusetzen und die dazu erforderliche Beseitigung der noch bestehenden strukturellen Hemmnisse zu befördern. Vor diesem Hintergrund lädt die DGIV zu ihrem Bundeskongress ein, der sich dem Themenkomplex CORONA (Lessons learned/ Resilienz) widmet.

\section{Lehren aus der CORONA-Krise für die Integrierte Versorgung \\ - CORONA-Krise - Katalysator zur Überwindung der Sektorengrenzen \\ - Integrierte Versorgung - CORONA und danach \\ - Ethische Erkenntnisse aus der CORONA- Krise}

\section{Sektorenübergreifende Versorgung}

- Bewertung der Arbeitsergebnisse der Bund-Länder-Arbeitsgruppe sektorenübergreifende Versorgung

- Zu wenig Freiheit oder zu viele GruppenEgoismen? - Woran hakt es bei der Weiterentwicklung der integrierten Selektivversorgung?

- Wieviel „ambulant“ können Krankenhäuser und wieviel "stationär" können Vertragsärzte leisten?
CORONA-Erfahrungen - die medizinische Perspektive

- CORONA-Erfahrungen aus erster Hand "Hotspot" Madrid

- CORONA-Erfahrungen aus der Hauptstadt Deutschlands - am Beispiel von Vivantes

- CORONA-Erfahrungen aus der Onkologie - wie sieht die Tumormedizin der Zukunft aus?

\section{Podiumsdiskussion „Wie sieht die Roadmap nach CORONA aus?"}

\section{Veranstalter}

DGIV e.V., Wartburgstraße 11, 10823 Berlin, Tel.: 030/44727080, Fax: 030/44729746,

E-Mail:info@dgiv.org

\section{Wissenschaftliche Leitung}

Prof. Dr. Stefan G. Spitzer, Dresden

Prof. Dr. Dr. Alfred Holzgreve, Berlin

Dr. Michael Meyer, Berlin

\section{Tagungsort}

Hotel Aquino, Tagungszentrum Katholische Akademie, Hannoversche Straße 5b, 10115 Berlin-Mitte

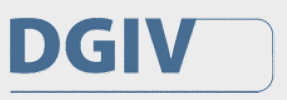

Deutsche Gesellschaft fii Integrierte Versorgung im Gesundheitswesen e.V.

Quelle und weitere Infos: www.dgiv.org 